\title{
PNEUMOMEDIASTINO ESPONTÂNEO - SÍNDROME DE HAMMAN: relato de caso
}

Fernando Augusto de Vasconcellos SANTOS ${ }^{1}$ João Cláudio Soares de SOUSA ${ }^{2}$ João Bernardo Sancio Rocha RODRIGUES ${ }^{3}$

Douglas Oliveira da $\operatorname{COSTA}^{4}$ Pedro Tolentino Figueiredo Guimarães SANTOS ${ }^{5}$ Mariana Brandão de OLIVEIRA ${ }^{6}$ Odail da Silva REZENDE JÚNIOR ${ }^{7}$

1 - Mestre em cirurgia pela Faculdade de Medicina da UFMG, Membro Titular da SBC, Coordenador da Residência Médica de Cirurgia Geral e Cirurgia do Aparelho Digestivo do Hospital Governador Israel Pinheiro - IPSEMG (e-mail: fernandoavsantos@gmail.com).

2 - Médico Cirurgião Geral e Residente de Cirurgia do Aparelho Digestivo do Hospital Governador Israel Pinheiro IPSEMG

(e-mail: joaoclaudiojoao@ hotmail.com).

3 - Médico Residente de Cirurgia Geral do Hospital Governador Israel Pinheiro - IPSEMG

(e-mail: joao.sancio@hotmail.com).

4 - Médico Residente de Cirurgia Geral do Hospital Governador Israel Pinheiro - IPSEMG (e-mail: dcostacosta@gmail.com).

5 - Médico Residente de Cirurgia Geral do Hospital Governador Israel Pinheiro - IPSEMG (e-mail: pedrotolentino1988@gmail.com).

6 - Médica Residente de Cirurgia Geral do Hospital Governador Israel Pinheiro - IPSEMG

(e-mail: mariana_didi@hotmail.com).

7 - Médico Residente de Cirurgia Geral do Hospital Governador Israel Pinheiro - IPSEMG

(e-mail: odailrezende@gmail.com).

\section{Recebido em: 08/02/2015 - Aprovado em: 13/06/2015 - Disponibilizado em: 15/07/2015}

Resumo - Pneumomediastino espontâneo é uma rara condição definida pela presença de ar livre no mediastino, na ausência de história recente de trauma, operações ou outros procedimentos invasivos. São considerados fatores desencadeantes quadros de vômitos incoercíveis, crises intensas de tosse, uso de drogas inalatórias, atividades físicas, broncoespasmo e até mesmo gritos intensos ou uso de instrumentos de sopro. Apresenta evolução benigna e autolimitada, sendo mais frequente em homens jovens. Clinicamente, sua apresentação usual inclui dor torácica, dispneia e enfisema subcutâneo. A seguir, relata-se o caso de um paciente do sexo masculino de 19 anos de idade, que se apresentou inicialmente com quadro de dor abdominal epigástrica de moderada intensidade, associada a vômitos incoercíveis após libação alcoólica. Recebeu tratamento sintomático, retornando após três dias com quadro de dor abdominal predominantemente em epigástrio, além de vômitos, desta vez associado a episódio de hematêmese e extenso enfisema subcutâneo em regiões cervical e torácica. Radiografia de tórax evidenciou presença de pneumomediastino além de pequeno pneumotórax bilateral. Tomografia computadorizada de tórax confirmou esses achados, revelando também a presença de pneumoraqui. Aventada hipótese de ruptura esofágica, excluída por esofagograma que não mostrou extravasamento de contraste. Por exclusão, caracterizou-se o diagnóstico de síndrome de Hamman. O paciente evoluiu sem intercorrências e com melhora clínica.

Palavras-chave: Enfisema Mediastínico. Diagnóstico de Pneumomediastino. Enfisema Subcutâneo. Dor Abdominal. Pulmão. 


\section{SPONTANEOUS PNEUMOMEDIASTINUM - HAMMAN'S SYNDROME: a case report}

Abstract - Spontaneous pneumomediastinum is a rare condition defined by the presence of air in the mediastinum, in
the absence of recent history of trauma, surgery or other invasive procedures. Are considered as triggering factors
severe emesis, intense attacks of coughing, use of inhalational drugs, physical activity, bronchospasm and even intense
screaming or playing of wind instruments. Is a condition that presents benign and self- limited evolution, which is more
common in young males. Clinically, its usual presentation includes chest pain, dyspnea and subcutaneous emphysema.
We report a case of a male patient of 19 years old, who initially presented with epigastric abdominal pain of moderate
intensity associated with uncontrollable emesis after alcoholic libation. Received symptomatic treatment, returning after
three days with abdominal pain predominantly in epigastrium, and emesis, this time associated with an episode of
hematemesis and extensive subcutaneous emphysema in the cervical and thoracic regions. Chest radiography
demonstrated the presence of pneumomediastinum and a small bilateral pneumothorax. Computed tomography
confirmed these findings, also revealing the presence of pneumoraqui. Considered the possibility of esophageal
perforation, excluded by barium swallow showed no contrast extravasation. By exclusion, the diagnosis of Hamman's
syndrome was characterized. The patient recovered uneventfully and with clinical improvement. Key-words: Mediastinal Emphysema. Pneumomediastinum, Diagnostic. Subcutaneous Emphysema. Abdominal pain. Lung.

\section{INTRODUÇÃO}

Pneumomediastino

espontâneo,

também conhecido como Síndrome de

Hamman, é rara condição definida pela

presença de ar livre no mediastino, na

ausência de história recente de trauma,

operações ou outros procedimentos invasivos

(IYER et al., 2009). Originalmente descrita por Louis Hamman em 1939, é conhecida por seu caráter benigno, sendo mais comum em adultos jovens expostos a aumentos bruscos da pressão da cavidade torácica, o que resulta em aumento da pressão intra-alveolar, seguido de sua ruptura e extravasamento de ar (CACERES et al., 2008). Vômitos, tosse, crises asmáticas, exercícios físicos, infecções das vias aéreas superiores e uso de drogas inalatórias, dentre outros, são alguns dos fatores predisponentes relacionados (MEIRELES et al., 2011).

\section{RELATO DO CASO}

Paciente do sexo masculino, de 19 anos de idade, foi admitido no serviço médico de urgência (SMU) do Hospital Governador Israel Pinheiro (HGIP) pela equipe de clínica médica com quadro de dor abdominal epigástrica de moderada intensidade, associado a vômitos incoercíveis após libação alcoólica durante a madrugada do dia anterior. Paciente relatou ainda consumo de maconha no dia anterior. Quanto à história pregressa, o paciente negava doenças associadas, alergias ou operações prévias. Referiu uso esporádico de cocaína. Ao exame físico, apresentava-se em bom estado geral, afebril, sem alterações hemodinâmicas ou respiratórias, com presença de dor abdominal moderada à palpação profunda de epigástrio, sem sinais de irritação peritoneal. Não foram notadas alterações da pele ou subcutâneo. Realizada hidratação venosa e antieméticos, evoluindo com melhora parcial da dor abdominal e 
interrupção dos vômitos. Paciente recebeu alta com orientações.

Passados três dias, o paciente foi readmitido com relato de retorno de quadro de dor abdominal predominantemente em epigástrio, além de vômitos persistentes, desta vez associado a episódio de hematêmese e aumento do volume cervical. Ao exame, encontrava-se estável do ponto de vista hemodinâmico, podendo ser notado extenso enfisema subcutâneo em região cervical e parte superior da região torácica anterior, com crepitações subcutâneas nesses locais. Os exames laboratoriais (hemograma, íons, função renal e proteína $\mathrm{C}$ reativa) não apresentavam alterações. Realizada radiografia de tórax (FOTO 1), na qual se observou sinais de enfisema subcutâneo difuso em parede torácica, discreto pneumotórax bilateral, além de sinais de pneumomediastino.
FOTO 1 - Radiografia de tórax. A: enfisema subcutâneo e pneumomediastino. B: em maior aumento, visibiliza-se discreto pneumotórax à esquerda (setas tracejadas) e ar no mediastino - dupla linha na silhueta cardíaca (setas contínuas).
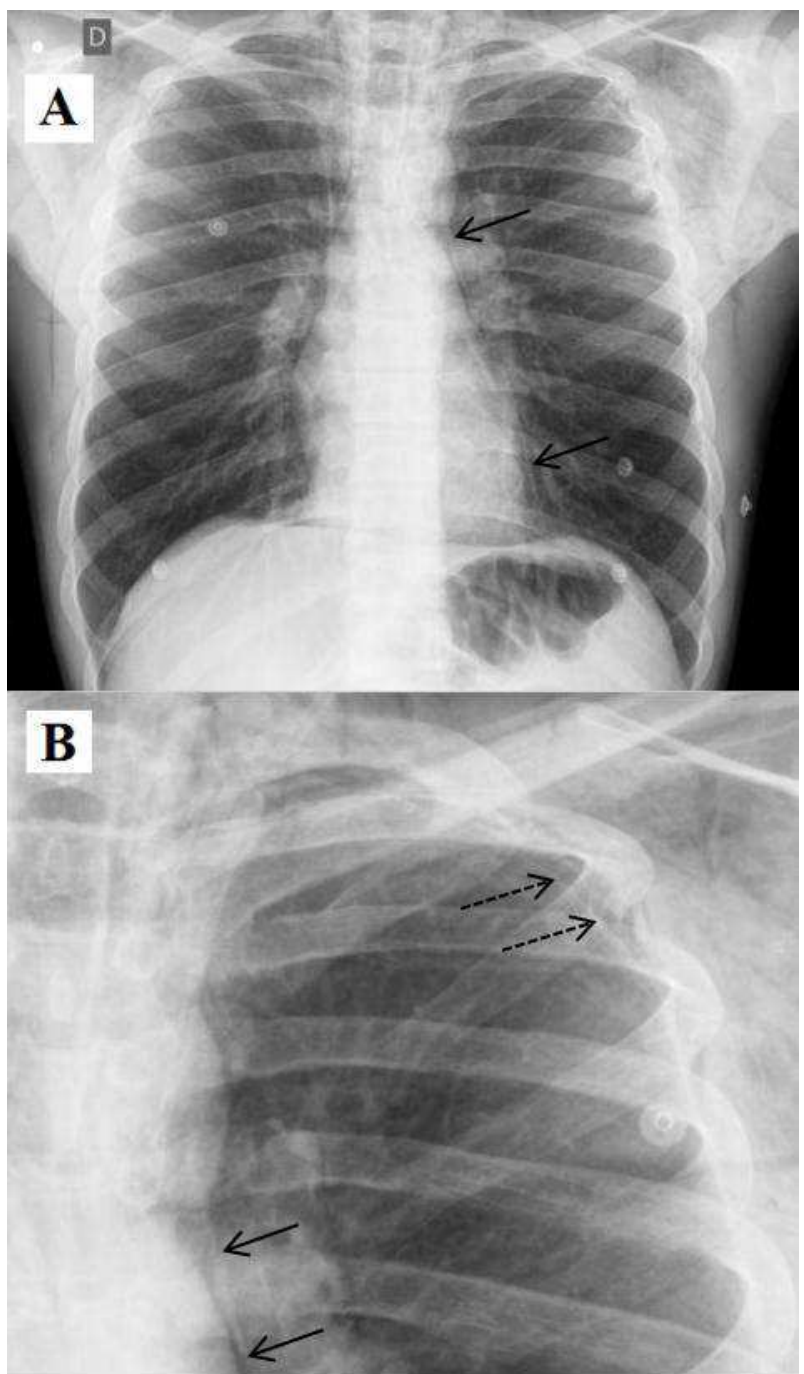

Fonte: Fotografia de exame realizado em nosso serviço. 
Solicitada avaliação da equipe de cirurgia geral sob a hipótese de ruptura esofagiana espontânea - Síndrome de Boerhaave, sendo mantido paciente em jejum e iniciado esquema antibiótico com Clindamicina e Ceftriaxone. Também foi optado por transferir o paciente para observação em leito de centro de tratamento intensivo (CTI) e realizar esofagograma (FOTO 2), no qual não se evidenciou extravasamento de contraste.
FOTO 2 - Esofagograma sem evidências de extravasamento de contraste - A: porção cervical. B: tórax e transição toracoabdominal.

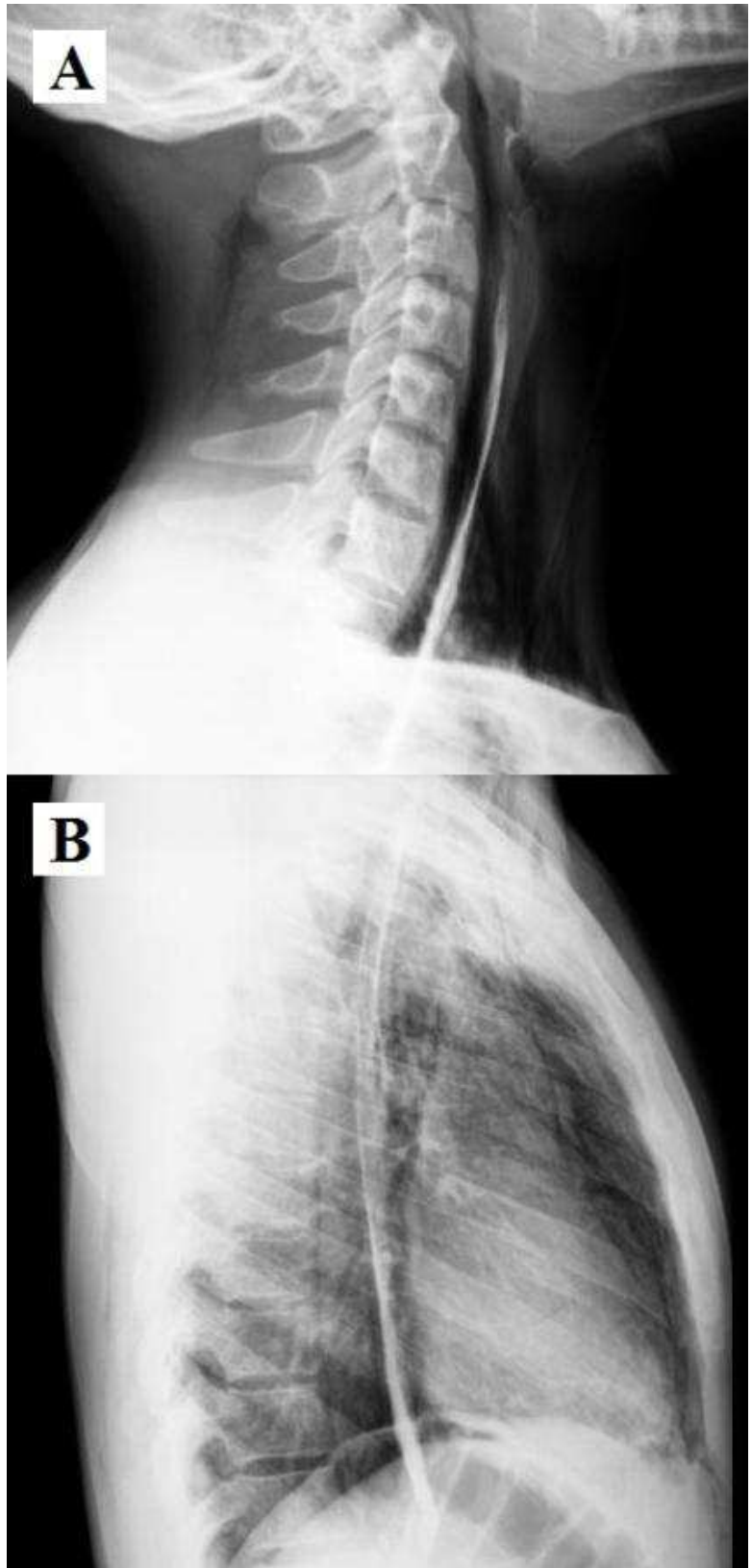

Fonte: Fotografia de exame realizado em nosso serviço. 
No segundo dia de internação, paciente se manteve sem novos episódios de vômitos ou hematêmese, com melhora da dor abdominal. Ao exame físico não apresentava novas alterações. Realizada tomografia computadorizada de tórax (FOTO 3), observando-se presença de ar dissecando os planos musculares e circundando as estruturas vasculares das regiões mediastinal, cervical, parede torácica anterior, axilas, espaços intercostais, além de ar no interior do canal medular (pneumorraqui). Não foi observado extravasamento de contraste oral nos terços médio e inferior do esôfago.

FOTO 3 - Tomografia de tórax contrastada evidenciando a presença de pneumomediastino, pneumorraqui e enfisema subcutâneo.

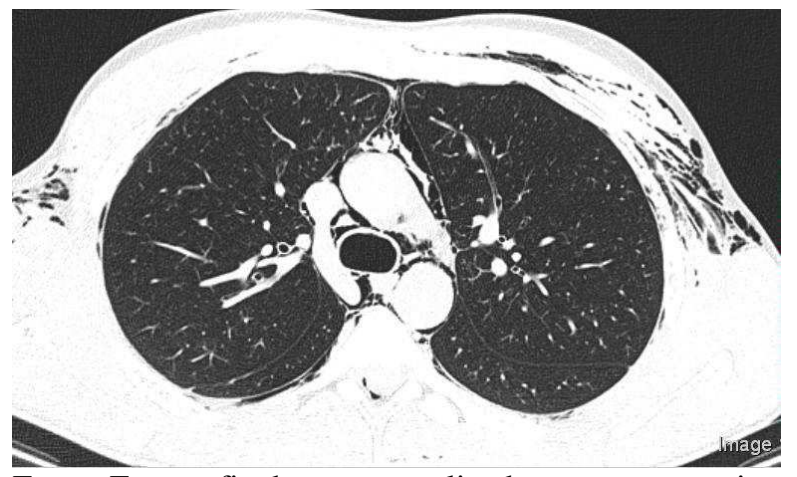

Fonte: Fotografia de exame realizado em nosso serviço.

Ao terceiro dia de internação foi liberada dieta oral, suspenso esquema antibiótico e liberada alta para enfermaria. O paciente evoluiu de maneira satisfatória, sem novas queixas, com melhora progressiva do enfisema subcutâneo, recebendo alta hospitalar após um total de seis dias de internação.

\section{DISCUSSÃO}

Pneumomediastino espontâneo é uma condição incomum e com poucos relatos na literatura (CACERES et al., 2008). Sua fisiopatologia foi delineada em estudo experimental elaborado por Macklin, em que se mostrou que o aumento súbito da pressão torácica, como ocorre durante manobras de Valsalva intensas, pode levar à ruptura alveolar, seguido do extravasamento e dissecção do ar pelo interstício e tecido bronco vascular do mediastino, podendo se estender para o tecido subcutâneo, espaço epidural, pericárdio e até mesmo cavidade peritoneal (IYER et al., 2009; CACERES et al., 2008; MEIRELES et al., 2011; ALMUFARREJ et al., 2008).

A maioria das séries de casos presentes na literatura descreve a presença de eventos desencadeantes para o desenvolvimento do pneumomediastino espontâneo, destacando-se os quadros de vômitos incoercíveis, intensas crises de tosse, o uso de drogas inalatórias, atividades físicas, broncoespasmo e até mesmo gritos intensos ou o uso de instrumentos de sopro. Entre estes, aqueles que são mais frequentemente tidos como precipitantes para a síndrome de Hamman são os quadros de vômitos, 
atividades físicas intensas, crises de tosse e gritos. Ainda assim, em mais de $30 \%$ dos casos, nenhum fator desencadeante pode ser identificado (CACERES et al., 2008). No presente relato, apesar da história associada de uso de droga inalatória de forma esporádica, pressupôs-se que o fator desencadeante mais provável foi o quadro de vômitos incoercíveis após libação alcoólica.

\section{Pneumomediastino pode ser} classificado em espontâneo, quando não há eventos prévios evidentes, como traumas ou intervenções que justifiquem a condição; ou secundário, este relacionado a eventos específicos, como perfurações esofágicas contidas, pequenas lacerações na árvore traqueobrônquica e infecções pulmonares ou mediastinais latentes (CACERES et al., 2008). Quando comparados os casos de pneumomediastino espontâneo com aqueles de origem secundária, nota-se que, apesar de ambos predominarem no sexo masculino, o pneumomediastino secundário está relacionado à maior necessidade de intubação oro traqueal durante sua condução (46\% versus 7\%), maior associação com pneumotórax (56\% versus $14 \%)$ e derrames pleurais (12\% versus $0 \%$ ), além de maior tempo de internação hospitalar (19 dias versus 3 dias) e maior mortalidade (39\% versus $0 \%$ ) (CACERES et al., 2008).

Esta síndrome é mais frequente em adultos jovens do sexo masculino, sendo a dor torácica - em sua maioria, retroesternal, o sintoma mais comumente encontrado, seguido de dispneia e tosse ou desconforto cervical (IYER et al., 2009; CACERES et al., 2008). As queixas de dor abdominal e enfisema subcutâneo cervical que levaram nosso paciente a retornar ao hospital foram encontradas em, respectivamente, $5,9 \%$ e 11,8\% dos casos estudados por Al-Mufarrej et al. (AL-MUFARREJ et al., 2008). Takada et al. encontraram enfisema subcutâneo em $68 \%$ dos pacientes incluídos em seu estudo (TAKADA et al., 2008). A presença de estertores crepitantes associados aos batimentos cardíacos - sinal de Hamman, é considerada sinal patognomônico desta condição, podendo ser encontrado, no entanto, em somente $10 \%$ a $20 \%$ dos casos (ALVES et al., 2012). Em nosso caso não foi notado sinal de Hamman.

Seu diagnóstico diferencial inclui condições associadas à dor torácica - seu principal sintoma, tais como síndrome coronariana aguda, pericardite, espasmo esofagiano, pneumotórax e tromboembolismo pulmonar, bem como causas de pneumomediastino secundário, como ruptura traqueobrônquica ou esofágica (CACERES et al., 2008; MEIRELES et al., 2011).

A síndrome de Boerhaave, suspeita diagnóstica inicial neste relato, apresenta algumas características semelhantes ao quadro de pneumomediastino espontâneo, como dor 
torácica e enfisema subcutâneo, além do fato de comumente surgir após episódios de vômitos repetidos. Entretanto, geralmente há notável elevação de marcadores inflamatórios, diferentemente do que costuma ocorrer na síndrome de Hamman, em que as alterações laboratoriais são, em sua maioria, discretas ou inexistentes. Além disso, sinais sistêmicos como febre, taquicardia e hipotensão são mais comuns na ruptura esofágica (MACIA et al., 2007). Sendo assim, na presença de sinais de inflamação grave em casos suspeitos de pneumomediastino espontâneo, deve-se, primeiramente, excluir a possibilidade de ruptura esofágica, haja vista que a mediastinite decorrente da mesma evolui com elevadas taxas de mortalidade caso não seja diagnosticada e tratada de forma precoce (TAKADA et al., 2008).

A radiografia de tórax é o principal exame utilizado como método diagnóstico, podendo ser evidenciada uma dupla linha delineando o mediastino (KOULLIAS et al., 2004). A incidência em perfil, além da póstero anterior, é de extrema importância, uma vez que estudos mostraram que mais de $50 \%$ dos diagnósticos podem ser perdidos quando realizada somente a incidência póstero anterior (MACIA et al., 2007). A tomografia computadorizada, padrão-ouro para detecção de ar no mediastino, é outro exame complementar que pode ser utilizado para confirmação diagnóstica, apresentando também grande importância para exclusão de outras causas ou condições associadas. O estudo contrastado do esôfago, a endoscopia digestiva alta e a broncoscopia ficam reservados para os casos de evolução arrastada, quando o diagnóstico é realizado de forma tardia, na presença de febre ou leucocitose associada, ou nos casos em que há suspeita de ruptura esofagiana ou traqueobrônquica (MEIRELES et al., 2011; KOULLIAS et al., 2004).

Apesar da ausência de maiores estudos que definam o manejo ideal dos pacientes com pneumomediastino espontâneo, a abordagem aceita pela maioria dos autores consiste em oxigenoterapia, analgesia e repouso (MACIA et al., 2007). A utilização de antibióticos profiláticos é controversa, sendo mais sensato seu uso nos casos em que há suspeita de mediastinite, ou até se concluir a propedêutica e serem excluídas outras condições (MEIRELES et al., 2011; KOULLIAS et al., 2004). De uma forma geral, os pacientes apresentam rápida recuperação, com tempo médio de internação de três dias, sendo raro o desenvolvimento de complicações ou recorrência (CACERES et al., 2008; KOULLIAS et al., 2004; GERAZOUNIS et al., 2003). 


\section{CONCLUSÃO}

O pneumomediastino espontâneo síndrome de Hamman é condição rara e benigna, mais frequente em homens jovens, que, em geral, evolui com rápida recuperação e infrequente recorrência. Sua apresentação clínica típica inclui dor torácica, dispneia e enfisema subcutâneo. Fatores desencadeantes que levam ao aumento brusco da pressão intratorácica muitas vezes podem ser identificados, sendo o quadro de vômitos repetidos considerado o mais comum. O diagnóstico, em sua maioria, é realizado por meio da radiografia de tórax, podendo ser confirmado com tomografia computadorizada do tórax. Estudos como esofagograma, endoscopia digestiva e broncoscopia ganham importância nos casos de evolução arrastada ou persistência de dúvida diagnóstica. Os principais diagnósticos diferenciais incluem causas de dor torácica, além de ruptura traqueobrônquica e esofágica. O manejo, apesar de não estar completamente definido, inclui o uso de oxigenoterapia, analgesia e repouso, sendo a utilização de antibióticos profiláticos controversa.

\section{REFERÊNCIAS}

AL-MUFARREJ, Faisal et al. Spontaneous pneumomediastinum: diagnostic and therapeutic interventions. Journal of
Cardiothoracic Surgery, v. 3, n. 3, p. 59, 2008.

ALVES, Giordano R. T. et al.

Pneumomediastino espontâneo (síndrome de Hamman). Jornal Brasileiro de

Pneumologia, v. 38, n. 3, p. 404-407, 2012.

CACERES, Manuel et al. Spontaneous pneumomediastinum: a comparative study and review of the literature. Annals of Thoracic Surgery, v. 86, n. 3, p. 962-966, 2008.

GERAZOUNIS, Michalis et al. Spontaneous pneumomediastinum: a rare benign entity. The Journal of Thoracic and Cardiovascular Surgery, v. 126, n. 3, p. 774-776, 2003.

IYER, V. N.; JOSHI, A. Y.; RYU, J. H. Spontaneous pneumomediastinum: analysis of 62 consecutive adult patients. Mayo Clinic Proceedings, v. 84, n. 5, p. 417-421, 2009.

KOULLIAS, George J. et al. Current assessment and management of spontaneous pneumomediastinum: experience in 24 adult patients. European Journal of Cardio- 
thoracic Surgery, v. 25, n. 5, p. 852-855, 2004.

MACIA, Ivan et al. Spontaneous pneumomediastinum: 41 cases. European Journal of Cardio-thoracic Surgery, v. 31, n. 6, p. 1110-1114, 2007.

MEIRELES, José et al. Spontaneous pneumomediastinum revisited. Respiratory

Medicine CME, v. 4, n. 4, p. 181-183, 2011.

TAKADA, Kazuto et al. Management of spontaneous pneumomediastinum based on clinical experience of 25 cases. Respiratory Medicine, v. 102, n. 9, p. 1329-1334, 2008. 